\title{
Effects of Multicomponent Exercise Training on Physical Functioning among Institutionalized Elderly
}

\author{
Maria Justine, ${ }^{1}$ Tengku Aizan Hamid,, ${ }^{2}$ ikram Mohan, ${ }^{1}$ and Madhanagopal Jagannathan ${ }^{3}$ \\ ${ }^{1}$ Physiotherapy Department, Faculty of Health Sciences, Universiti Teknologi MARA, Puncak Alam Campus, \\ 42300 Puncak Alam, Selangor, Malaysia \\ ${ }^{2}$ Institute of Gerontology, Universiti Putra Malaysia, 43400 Serdang, Selangor, Malaysia \\ ${ }^{3}$ School of Physiotherapy, Faculty of Allied Health Professions, AIMST University, 08100 Bedong, Kedah, Malaysia \\ Correspondence should be addressed to Maria Justine, maria205@salam.uitm.edu.my
}

Received 1 July 2011; Accepted 14 August 2011

Academic Editors: B. Murphy, M. Pääsuke, and J. J. Sosnoff

Copyright ( $) 2012$ Maria Justine et al. This is an open access article distributed under the Creative Commons Attribution License, which permits unrestricted use, distribution, and reproduction in any medium, provided the original work is properly cited.

This quasiexperimental study aimed to measure the effects of 12-week multicomponent exercise training on physical functioning among institutionalized elderly. Forty-three participants (age $=70.88 \pm 7.82$ years) were self-assigned to an intervention $(n=23)$ or control $(n=20)$ group. Before and after training, cardiorespiratory endurance, arm curl strength, grip strength, lower limb strength, upper and lower limb flexibility, balance, and mobility were assessed. The training included aerobic, resistance, balance and flexibility exercises, performed 3 times per week. Significant increases $(P<0.05)$ were observed in the exercise group on cardiorespiratory endurance (41.79\%), right arm curl strength (25\%), left arm curl strength (30.79\%), right hand grip strength (13.65\%), left hand grip strength (9.93\%), lower limb strength (46.19\%), balance $(49.58 \%)$, and mobility (26.37\%). Measures of flexibility in the exercise group also showed improvement (right lower limb (63.57\%), left lower limb (44.17\%), right upper limb (36.67\%), and left upper limb (63.1\%)) but were not statistically significant (all, $P>0.05$ ). The control group did not show any significant changes $(P>0.05)$ in any variables. The data suggested that 12 -week multicomponent exercise training may improve physical functioning among institutionalized elderly.

\section{Introduction}

Physical functioning is widely recognized as a crucial component of quality of life (QOL) and perhaps the most universally accepted indicator of health status in older persons [1]. Physical functioning that comprises muscle strength, flexibility, balance, agility, and aerobic endurance is also known as functional fitness. It has been defined as having the capacity to perform day-to-day activities safely and independently without fatigue [2-4]. Aging leads to a decline in physical functioning [1] that causes a high level of dependency $[5,6]$. Improving physical functioning or functional fitness components separately or in combination enables older adults to maintain a range of functional movement, such as bathing, toileting, climbing stairs, rising out of chair, walking, and/or using a wheelchair so that they can adopt a more active lifestyle and thus promotes their QOL.

A number of recommendations suggest that physical activity should focus on improving functional fitness by engaging in cardiorespiratory, flexibility, strength, and balance training 3 to 5 days per week [7-9]. As such, in order to preserve physical functioning, it is proposed in this study that exercise training should include aerobic, strengthening, flexibility, and balance training. Previous studies on exercise interventions for institutionalized older persons mainly emphasized one or two fitness components such as strength alone or a combination of strength and aerobic training. Aerobic and strength training had been shown to operate through different mechanisms in promoting health in the older persons [10]. Strength training improves muscle mass, muscle strength, muscle function, and balance [11-14] while aerobic training mainly alters cardiovascular fitness, blood pressure, and plasma lipoprotein profiles $[15,16]$, but with no improvement in flexibility. More recently, it has been identified that balance is a key element in exercise programs aiming to reduce the falls risk among older persons [17].

According to the American College of Sports Medicine (ACSM) and other literatures, recommendation for exercise 
interventions should consist of aerobic activities, strength training, balance, and flexibility [7, 18-25]. However, the majority of available information about the effectiveness of exercise on strength, balance, cardiovascular fitness, and flexibility domains has been conducted among communitydwelling older persons with limited evidence from institutionalized older persons. Furthermore, there is lack of documentation on the effectiveness of multicomponent exercise program on physical functioning among institutionalized older persons. Hence, the purpose of this study was to measure the effects of 12-week multicomponent exercise training on physical functioning among institutionalized elderly.

\section{Methods}

2.1. Subjects and Experimental Design. Forty-three institutionalized elderly $($ female $=21$, male $=22$ ) aged above 60 years old volunteered to participate in this quasiexperimental study. The inclusion criteria were as follows: able to walk six meters or more with or without any walking devices, free from medical conditions that would prohibit safe participation in low to moderate intensity exercise independently, able to comprehend the study procedures and instructions, not currently involved in a regular exercise program of $>30$ minutes per day for three times a week, and independent in basic activities of daily living. The purpose and design of the study were fully explained to each subject before they gave written informed consent. The study was approved by the Ethics committee of the Faculty of Medicine, Universiti Putra Malaysia.

2.2. The Exercise Group. The multicomponent exercise training was conducted 3 times per week on every Monday, Wednesday, and Friday over a period of 12 weeks, and each session lasted for 1 hour. The exercises were conducted in the physiotherapy department by a physiotherapist with a staff nurse on standby to assist the older persons in case of any emergency.

The exercise program consisted of: (1) Five to 10 minutes of warm-up exercise, including mobilizing exercises (neck, trunk, upper and lower limb) and breathing exercises; (2) Aerobic exercises involving upper and lower limbs alternately in seated position, starting at low intensity (40-50\% maximal heart rate) for first 2 weeks for 20 minutes, progressed to moderate intensity of exercise at slight breathlessness which is equivalent to $50-70 \%$ of maximal heart rate [6] for 20 to 45 minutes; (3) balance exercises included were lifting one leg forward, backward, sideways, tip toeing, and heelstanding while moving the arms forward and backward rhythmically and continuously; (4) strengthening exercises were performed with elastic band, twice a week on every Monday and Friday. In the first two weeks of the program, the subjects performed exercise for upper and lower limb muscles alternately at a very low intensity of 1 to 3 sets. Each set consists of 5 to 8 repetitions, 1 minute rest given between each set. The duration for the strengthening exercise was between 15 and 20 minutes. The resistance of the elastic band was determined by asking each subject to stretch the elastic band to double the initial length for 10 repetitions without feeling fatigue. After 2 weeks of training, each exercise was progressed between 2 and 4 repetitions at every 3 weeks, until the subjects complete 15 repetitions; (5) stretching exercises were performed for major muscle groups of the upper limb (flexors, extensors, abductors, adductors of arm, and forearm muscles) and lower limb (hip flexors, extensors, abductors and adductors, knee extensors, dorsiflexors and plantarflexors). Each stretch was sustained between 15 and 30 seconds with 1 to 3 repetitions for each muscle [26]; (6) at the end of each session, 5 to 10 minutes cool-down period involving mobilizing exercise (neck, trunk, and upper and lower limb) and breathing exercise were performed.

2.3. The Control Group. The subjects in the control group received a session of briefing on health education, at the beginning of the study. The education session highlighted the importance of regular physical activity and exercise. Subjects were followed up by the researcher once in two weeks and advised to continue with their previous lifestyle.

2.4. Outcome Measures. The variables measured in this study included cardiorespiratory or aerobic endurance, muscular function, flexibility, balance, and mobility. The instruments to measure the outcome of the intervention had been described as practical, valid, and reliable for older person $[4,27]$.

Aerobic endurance was measured using the 6 min walk test. The subject was asked to walk as fast as possible for 6 minutes throughout the test. The score was the total distance walked in 6 minutes along a $45.72 \mathrm{~m}$ rectangular course, which was marked in every $4.57 \mathrm{~m}$.

The chair stand test was used to assess the limb lower muscle endurance particularly the knee extensor. The subject was instructed to sit on a firm straight-backed chair (height approximately $17^{\prime \prime}$ ) with arms folded across the chest with arms remained in this position for the entire test. The subject was instructed to rise to a full stand and return back to a fully seated position after the signal "go" was given. The subject was encouraged to complete as many full stands as possible within a 30-second time. After a demonstration by the researcher, the subject completed a practice trial of two repetitions, followed by one 30 -second test trial. The number of stands performed within 30 seconds was recorded as the subject's score for the lower limb muscle endurance.

The grip strength was measured using a hand dynamometer (Lafayette Instrument J00105 JAMAR Hydraulic Hand Dynamometer). The subject was asked to hold the dynamometer in the hand, with the shoulder adducted, elbow flexed to 90 degrees, forearm pronated, slight wrist extension, and ulnar deviation position. The subject was instructed to squeeze the dynamometer with maximum isometric effort, maintained for about 2 to 5 seconds. The researcher demonstrated the procedure before the test was conducted, and then the subject performed three test trials with 60 -second rest given between trials. The average of the three readings was recorded as grip strength.

The arm curl endurance was measured based on the number of arm curl movement in 30 seconds using a dumbbell [4]. The subject was asked to sit at the edge of the 
TABLE 1: Demographic characteristics of study population at baseline.

\begin{tabular}{|c|c|c|c|}
\hline Characteristics $(N=43)$ & Exercise $(n=23)$ & Control $(n=20)$ & $P$ value \\
\hline Mean age, years $(S D)$ & $70.19(8.84)$ & $71.80(6.88)$ & .506 \\
\hline Female, $n(\%)$ & $11(47.8)$ & $10(50.0)$ & - \\
\hline Male, $n(\%)$ & $12(52.2)$ & $10(50.0)$ & - \\
\hline Mean height, m $(S D)$ & $1.53(0.08)$ & $1.53(0.07)$ & .979 \\
\hline Mean weight, kg (SD) & $53.18(9.11)$ & $55.30(10.34)$ & .480 \\
\hline Mean BMI, $\mathrm{kg} / \mathrm{m}^{2}(S D)$ & $22.93(4.17)$ & $23.95(5.23)$ & .479 \\
\hline \multicolumn{4}{|l|}{ Ethnicity } \\
\hline Malay & $14(60.9)$ & $13(65.0)$ & - \\
\hline Chinese & $5(21.7)$ & $4(20.0)$ & - \\
\hline Indian & $3(13.0)$ & $3(15.0)$ & - \\
\hline Others & $1(4.3)$ & - & - \\
\hline Length of residency, years $(S D)$ & $3.14(2.82)$ & $4.56(3.97)$ & .165 \\
\hline
\end{tabular}

chair to ensure full range of motion of elbow joint. Then, the subject was instructed to do flexion and extension of elbow joint with $5 \mathrm{lb}$ weight for females and $8 \mathrm{lb}$ for males in supinated forearm within 30 seconds. After a demonstration by the researcher, the subject completed a practice trial of two repetitions, followed by one 30 -second test trial. The number of arm curl performed within 30 seconds was recorded as arm curl strength.

The chair sit-and-reach test (CSR) was used to assess lower limb flexibility particularly of the hamstrings and lower spinal extensors. Subjects were asked to sit on the edge of a chair; one leg was flexed at 90 degrees with the foot flat on the floor while the other leg was extended straight in front of the hip with heel on the floor and foot flexed at 90 degrees. The subject was instructed to bend forward at the hip while sliding hands down (overlapping each other) the extended leg as far as possible, holding the position for 2 seconds and the distance was measured from the middle fingers to the toes using a ruler. This was repeated 3 times, and the best of the 3 distances was taken.

The back scratch test (BS) was used to assess the upper limb flexibility [4]. The subjects were seated on the side of a chair; one arm was flexed beyond the shoulder with elbow flexed to the maximum, while the other arm extended and internally rotated with both the index fingers attempting to touch each other than holding for 2 seconds. The distance was measured using a ruler that the subject was short of reaching both the middle fingers (minus score) or beyond the middle fingers (plus score). This was repeated 3 times, and the best of the 3 distances was taken. Both the left and right upper limbs were measured with the top arm indicating the side to be measured.

The functional reach test (FRT) was used to measure balance that is required for postural control during perturbed movement $[28,29]$. The balance was measured as described by Duncan et al. [28]. The researcher recorded the functional reach in inches for all three trials and then averaged the three trials.

The time up-and-go test (TUG) was used to measure functional mobility [30]. The subject was instructed to stand up from a chair, walked a distance of 3 meters, turned around a cone, and returned to chair. The time taken to complete the test was measured by using stop watch. During the trial, the subject was required to wear an appropriate foot wear and those with walking devices were allowed to use them during the procedure.

2.5. Data Analysis. Descriptive statistics were performed on demographic characteristics, baseline and post-intervention results and data were reported as means $(\mathrm{M}) \pm$ standard deviations (SD). Baseline and posttest data were found to be normally distributed using Kolmogrov and Shapiro tests. Improvements were reported in percentage for all physical functioning tests. A two-way repeated measures ANOVA were used to compare differences between groups. The within-group changes between baseline and posttest were determined using paired $t$-tests. All statistical calculations were completed using the SPSS software (version 17.0), and level of significance was set at $P<0.05$.

\section{Results}

The demographic data for the subjects are illustrated in Table 1. No significant difference was found between the groups at baseline in relation to age, height, body weight, body mass index, and length of residency.

Table 2 presents the mean and standard deviation during baseline and posttest, and the percentage of changes in both the exercise and control groups.

The results of repeated measure ANOVA for cardiorespiratory endurance for both intervention and control groups revealed that there was no significant difference between groups after the 12-week duration $(F(1,41)=.778, P>0.05)$. Paired $t$-tests showed significant changes only in the exercise group $(P<0.05)$ but not in the control group $(P>0.05)$.

In terms of muscular function, there were no significant differences between groups after 12-week duration for measures of right arm curl $(F(1,41)=.374, P>0.05)$, left arm curl $(F(1,41)=1.292, P>0.05)$, right handgrip $(F(1$, $41)=0.092, P>0.05)$, and left handgrip $[F(1,41)=.400$, 
TABLE 2: Means, SD and \% changes in all measures before and after intervention for the exercise and control groups.

\begin{tabular}{|c|c|c|c|c|c|c|}
\hline \multirow{2}{*}{ Variables } & \multicolumn{3}{|c|}{ Exercise group $M \pm S D$} & \multicolumn{3}{|c|}{ Control group $M \pm S D$} \\
\hline & Baseline & 12-week & $\%$ change & Baseline & 12-week & $\%$ change \\
\hline CRE (m) & $208.92 \pm 87.62$ & $296.22 \pm 110.53$ & $41.79^{*}$ & $238.26 \pm 130.73$ & $213.11 \pm 129.70$ & -10.56 \\
\hline RAC (rep) & $12.91 \pm 4.92$ & $16.13 \pm 6.18$ & $24.95^{*}$ & $13.83 \pm 4.25$ & $13.15 \pm 3.25$ & -4.92 \\
\hline LAC (rep) & $13.04 \pm 5.50$ & $17.04 \pm 5.20$ & $30.7^{*}$ & $13.10 \pm 5.39$ & $12.65 \pm 4.22$ & -3.44 \\
\hline RHG (kg) & $14.61 \pm 7.86$ & $16.58 \pm 8.10$ & $13.55^{*}$ & $15.59 \pm 11.59$ & $16.63 \pm 9.14$ & 6.67 \\
\hline LHG $(\mathrm{kg})$ & $13.33 \pm 6.87$ & $15.99 \pm 7.29$ & $19.93^{*}$ & $15.67 \pm 8.13$ & $15.47 \pm 8.88$ & -1.29 \\
\hline LL (rep) & $9.22 \pm 4.04$ & $13.48 \pm 3.23$ & $46.19^{*}$ & $9.40 \pm 4.06$ & $9.05 \pm 3.52$ & -3.72 \\
\hline $\operatorname{RLL}(\mathrm{cm})$ & $-5.27 \pm 11.42$ & $-1.92 \pm 8.08$ & 63.57 & $-5.07 \pm 11.16$ & $-6.01 \pm 11.32$ & 18.54 \\
\hline $\operatorname{LLL}(\mathrm{cm})$ & $-3.78 \pm 10.23$ & $-2.09 \pm 7.81$ & 44.71 & $-4.56 \pm 9.40$ & $-5.30 \pm 9.68$ & 16.23 \\
\hline RUL (cm) & $-8.10 \pm-18.56$ & $-11.07 \pm 14.19$ & 36.67 & $-15.67 \pm 15.79$ & $-17.32 \pm 12.78$ & 10.53 \\
\hline LUL (cm) & $-12.48 \pm 19.9$ & $-20.35 \pm 14.77$ & 63.1 & $-21.12 \pm 11.91$ & $-22.04 \pm 10.68$ & 4.36 \\
\hline BAL (in) & $7.06 \pm 2.28$ & $10.56 \pm 2.64$ & $49.58^{*}$ & $7.13 \pm 2.47$ & $6.62 \pm 2.17$ & -7.15 \\
\hline MOB (sec) & $13.69 \pm 5.95$ & $10.08 \pm 3.75$ & $26.37^{*}$ & $13.59 \pm 6.78$ & $14.83 \pm 7.66$ & 9.12 \\
\hline
\end{tabular}

Note: CRE: cardiorespiratory endurance, RAC: right arm curl strength, LAC: left arm curl strength, RHG: right handgrip strength, LHG: left handgrip strength, LL: lower 1 limb strength, RLL: right lower limb flexibility, LLL: left lower limb flexibility, RUL: right upper limb flexibility, LUL: left upper limb flexibility, BAL: balance, MOB: mobility.

* Significance at 0.05 level.

$P>0.05]$. However, function of the lower limb muscles specifically the knee extensors differed significantly between the two groups after 12 -week duration $(F(1,41)=4.455$, $P<0.05)$. Paired $t$-tests showed significant changes only in the exercise group in all measures for the muscular function $(P<0.05)$.

There were no statistical differences between the two groups for measure of right lower limb flexibility $(F(1,41)=$ $.218, P>0.05)$, left lower limb flexibility $(F(1,41)=.611$, $P>0.05)$, as well as for right upper limb flexibility $(F(1$, $41)=2.241, P>0.05)$ and left upper limb flexibility $(F(1$, $41)=.906, P>0.05)$. There were no significant changes in the within-group paired $t$-tests for all measures of flexibility for both exercise and control groups (all, $P>0.05$ ).

Balance measure was found significantly different between the two groups after 12 weeks $(F(1,41)=8.198, P<$ $0.05)$ but not mobility $(F(1,41)=2.182, P>0.05)$. The within-group paired $t$-tests only showed significant changes in the exercise group for both balance and mobility measures $(P<0.05)$.

\section{Discussion}

4.1. Cardiorespiratory Endurance. The finding in cardiorespiratory endurance was consistent with previous RCT conducted among institutionalized older persons [31-33]. The present study showed improvement in 6MWT by $41.79 \%$ $(P<0.05)$ compared to only $8.1 \%(P<0.05)$ in a study carried previously [31] who conducted an exercise program twice a week for 15 minutes per session. In this study, it was found that greater improvement in 6MWT which could be due to the training protocol. The other possible explanation could be due to the improvement of the lower limb strength in the exercise group. This findings were supported by Bastone and Filho [31], who found significant correlation between cardiorespiratory endurance and lower limb strength $(r=0.517, P<.01)$ during baseline measurement. Studies also had shown from multivariate linear regression models that impairments in leg strength and power, especially those at the knee and ankle, were predictive of 6MWT performance [34].

The subjects in the exercise group started the cardiorespiratory exercise in the sitting position in the first two weeks and progressed to standing. In the standing position, the movements involved walking on the spot, stepping backward, forward, sideways, and lifting the knees with movements of the arms. These activities could have facilitated the movement while carrying out the 6MWT in which it involved walking at their own pace. The exercises were started in the sitting position to develop confidence and were also meant to familiarize subjects with various movements as there were many changes of movement throughout the exercise session.

4.2. Muscular Function. Changes in the muscular function were consistent with previous studies conducted to improve muscle strength and hand grip strength in older persons' institutions using RCT [31, 35-39]. The improvement in knee strength $(46.19 \%)$ was similar to a study conducted by Lazowski et al. [37] who found 55\% improvement in the knee strength among subjects who performed functional fitness training.

Intensity of the exercise selected in this present study was from low to moderate in order to reduce adverse effect. This was well supported in an RCT (exercise versus control) by Littbrand et al. [40] among older people (mean age = $85.3 \pm 6.1$ years) living in residential care facilities who were dependent in personal activities. The exercise group who performed high intensity training presented with 179 adverse events that include musculoskeletal (pain and soreness) 
(53\%), dizziness or discomfort from the chest (18\%), general unspecified (e.g., stomach pain) (4\%), psychological (e.g., fear of falling) (3\%), and near fall accident (1\%).

The protocol of the exercise to train the strength of the lower limb in this study was based on functional tasks of older persons. Previous studies that trained the lower limbs using a leg press machine did not produce significant improvement on knee strength $[32,41]$. The reason behind the insignificant improvement in their study is that the exercise performed in leg press machine is not consistent with the assessment of muscle strength using chair stand test. Therefore, it was decided in this study to train the older persons with movement such as half squatting and sitting to standing and progressing to holding objects that seems more functional which is normally required in their daily activity. The significant finding on the measure of the lower limb function is also attributable to the method of testing the outcome that is the 30 -second chair stand. This is considered relevant because the movement itself is considered functional and is required in daily function. As suggested by Kauffman et al. [42], functional outcomes are related to strength and motor performance and should be the objective of rehabilitation rather than muscle hypertrophy.

It was also observed in this study that the rate of change in the improvement of muscle function was not consistent which was at $46.19 \%, 24.95 \%, 30.7 \%, 13.55 \%$, and $19.93 \%$ for lower limb, right arm curl, left arm curl, right handgrip, and left handgrip, respectively. This is consistent with the argument by Gleberzon and Anis [43], who stated that, for a person who is aged, sarcopenia did not occur uniformly throughout the musculoskeletal system; certain muscles were negatively affected while others were relatively preserved. They added that quadriceps muscle atrophied to a much lesser extent than the biceps of the upper limb, which could be attributed to the fact that the quadriceps muscles were usually involved in everyday activity such as walking.

4.3. Flexibility. The observed findings of flexibility were not significant in the exercise group and are consistent with previous studies. Chin et al. [41] found only significant improvement in flexibility measured with the CSR in subjects performing functional training but not in the subjects performing strength only and combination of strength and functional training. Clearly, as in this study, when strength training was incorporated as part of the protocol, the changes in flexibility did not reach significant changes.

The possible reason for the lack of improvement in flexibility is duration of sustaining the stretch. In this study, the protocol was to stretch all major muscle groups for 15 to 20 seconds for 5 times every session as part of warm-up and cool-down movement. Previous studies which included stretching alone showed that 15 -second to 60 -second stretch enhanced flexibility of the lower limb $[26,44,45]$.

Longer holding times during stretching and daily performance might result in higher rate of gains in flexibility and a more sustained increase in flexibility in older persons. However, this was quite controversial as prolonging stretching causes increase in muscle length, and thus decreases the amount of passive force that it develops at certain points in the range of motion that the muscle crosses [46]. This finding has implications to the importance of regular stretching to maintain the length of the muscle rather than increasing the length of the muscle fibers which could reduce the tension necessary for stability of certain joints. Shortened muscle may help older persons in performing certain activities [46], as it maintains stability by providing tension to the joint.

4.4. Balance and Mobility. The results of functional mobility in this study were consistent with previous findings on older persons living in an institution [35]. Studies that had incorporated strength training to either aerobic, balance, or flexibility training have also shown significant improvement in mobility $[37,47,48]$. The statistically significant results for the balance measures could be due to the stimulation of proprioceptors in the joints which promoted stability or balance. Another reason that could be related to the balance might be due to the improvement of strength in the lower limb muscles.

The training in this study was incorporated with strength training, and the results in the measure of lower limb strength were significant in the exercise group. Thus, it can be concluded that it is associated with the improvement of the lower limb strength, as the relationship between leg strength and dynamic balance prior $(r=-.794, P=.01)$ and after $(r=-.790, P<.01)$ the training was significant. This is consistent with previous findings that muscle strength and power were related to balance performance among older persons [49-51]. In another study, Ringsberg et al. [52] revealed that older persons with good muscular strength in the legs had better balance than those with weaker legs. Daubney and Culham [53] argued that the force-generating capability of lower extremity muscle is important in the maintenance of balance. Clearly, interventions that have included strength training, together with balance training, showed significant improvement in balance rather than single intervention, such as only walking (cardiorespiratory training) or only flexibility training [37, 47].

It is reasonable to believe that short-term studies conducted over a few weeks have certain limitations. According to the American College of Sports Medicine [46], sedentary older subjects may take several weeks to adapt to the initial rigors of training and thus need a longer adaptation period to get the optimal benefit from a program. Thus, a longer duration of study is needed in order to determine effects especially on the level of psychological wellbeing.

4.5. Conclusion and Recommendation. The exercise program incorporated all the components of fitness required by older persons, which included strength, aerobic, flexibility, and balance training, and based on low frequency, low to moderate intensity, and using easily administrable and costeffective equipment. Each activity or movement was implemented to mimic functional tasks in order to train functions required in a real-life situation that is directed towards activities of daily living by means of sitting and standing.

The findings of this study support that exercise interventions which incorporate the elements of multicomponent exercise training improved cardiorespiratory endurance, 
muscle strength, and balance performance in institutionalized older persons. This shows that the majority of the frail older persons responded to the multicomponent exercise intervention. Therefore, the key clinical strategy is to support interventions that improve or maintain physical function. Thus, this might offer health care providers and practitioners the opportunity to develop exercise intervention which comprised the elements of multicomponent exercise training.

However, it is inconclusive as to which of the component of exercise was the actual cause of the outcome observed as the intervention used was a combination of cardiorespiratory, muscle strength, flexibility, and balance training. It is also not known that how much dosage or intensity of each component of this training had actually contributed to the improvement of each of the outcome that was observed. As such, future study is needed to compare a multicomponent exercise program with a single component of exercise intervention, for example, comparing a multicomponent exercise with aerobic training alone or with a combination of aerobic and strength training.

\section{Conflict of Interests}

None of the authors have conflict of interests to disclose.

\section{Acknowledgments}

Acknowledgement goes to Faculty of Health Sciences, Universiti Teknologi MARA for providing research materials for the study. The authors also wish to thank the participants of this study for their valuable contribution and time spent in the intervention.

\section{References}

[1] R. Kawamoto, O. Yoshida, and Y. Oka, "Factors related to functional capacity in community-dwelling elderly," Geriatrics and Gerontology, vol. 4, no. 2, pp. 105-110, 2004.

[2] K. Collins, B. L. Rooney, K. J. Smalley, and S. Havens, "Functional fitness, disease and independence in communitydwelling older adults in Western Wisconsin," Wisconsin Medical Journal, vol. 103, no. 1, pp. 42-48, 2004.

[3] W. L. Haskell and M. Kiernan, "Methodologic issues in measuring physical activity and physical fitness when evaluating the role of dietary supplements for physically active people," American Journal of Clinical Nutrition, vol. 72, no. 2, pp. 541s550s, 2000.

[4] C. J. Jones and R. E. Rikli, "Measuring functional fitness of older adults," The Journal on Active Aging, vol. 1, no. 2, pp. 2430, 2002.

[5] A. Noro and S. Aro, "Health-related quality of life among the least dependent institutional elderly compared with the noninstitutional elderly population," Quality of Life Research, vol. 5, no. 3, pp. 355-366, 1996.

[6] A. M. S. Singh, M. J. M. Chin, R. J. Bosscher, and W. V. Mechelen, "Cross-sectional relationship between physical fitness components and functional performance in older persons living in long-term care facilities," BMC Geriatrics, vol. 6, article 4, 2006.

[7] M. E. Nelson, W. J. Rejeski, S. N. Blair et al., "Physical activity and public health in older adults: recommendation from the American college of sports medicine and the American heart association," Medicine and Science in Sports and Exercise, vol. 39, no. 8, pp. 1435-1445, 2007.

[8] R. Topp, M. Fahlman, and D. Boardley, "Healthy aging: health promotion and disease prevention," Nursing Clinics of North America, vol. 39, no. 2, pp. 411-422, 2004.

[9] U.S. Department of Health and Human Services (USDHHS), Physical Activity and Health: A Report of the Surgeon General Executive Summary, U.S. Department of Health and Human Services, Centers for Disease Control and Prevention, National Center for Chronic Disease Prevention and Health Promotion, Atlanta, Ga, USA, 1996.

[10] D. J. Madden, J. A. Blumenthal, P. A. Allen, and C. F. Emery, "Improving aerobic capacity in healthy older adults does not necessarily lead to improved cognitive performance," Psychology and aging, vol. 4, no. 3, pp. 307-320, 1989.

[11] C. Barrett and P. Smerdely, "A comparison of communitybased resistance exercise and flexibility exercise for seniors," Australian Journal of Physiotherapy, vol. 48, no. 3, pp. 215-219, 2002.

[12] R.C. Cassilhas, V. A. R. Viana, V. Grassman et al., “The impact of resistance exercise on the cognitive function of the older persons," Medicine and Science in Sports and Exercise, vol. 39, no. 8, pp. 1401-1407, 2007.

[13] M. E. Nelson, M. A. Fiatarone, C. M. Morganti, I. Trice, R. A. Greenberg, and W. J. Evans, "Effects of high-intensity strength training on multiple risk factors for osteoporotic fractures: a randomized controlled trial," Journal of the American Medical Association, vol. 272, no. 24, pp. 1909-1914, 1994.

[14] R. Seguin and M. E. Nelson, "The benefits of strength training for older adults," American Journal of Preventive Medicine, vol. 25, no. 3, pp. 141-149, 2003.

[15] E. Rydwik, K. Frandin, and G. Akner, "Effects of physical training on physical performance in institutionalized elderly patients $(70+)$ with multiple diagnoses: systemic review," Journal of Age and Aging, vol. 33, no. 1, pp. 13-23, 2004.

[16] N. Takeshima, N. L. Rogers, M. E. Rogers, M. M. Islam, D. Koizumi, and S. Lee, "Functional fitness gain varies in older adults depending on exercise mode," Medicine and Science in Sports and Exercise, vol. 39, no. 11, pp. 2036-2043, 2007.

[17] C. Sherrington, J. C. Whitney, S. R. Lord, R. D. Herbert, R. G. Cumming, and J. C. T. Close, "Effective exercise for the prevention of falls: a systematic review and meta-analysis," Journal of the American Geriatrics Society, vol. 56, no. 12, pp. 2234-2243, 2008.

[18] American College of Sports Medicine, "Exercise and physical activity for older adults," Medicine and Science in Sports and Exercise, vol. 30, no. 6, pp. 992-1008, 1998.

[19] S. R. Bird, A. Smith, and K. James, Exercise Benefits and Prescription, Stanley Thornes Publishers, Cheltenham, UK, 1998.

[20] J. M. Heath and M. R. Stuart, "Prescribing exercise for frail elders," Journal of the American Board of Family Practice, vol. 15, no. 3, pp. 218-228, 2002.

[21] R. S. Mazzeo and H. Tanaka, "Exercise prescription for the elderly: current recommendations," Sports Medicine, vol. 31, no. 11, pp. 809-818, 2001.

[22] A. Y. McDermott and H. Mernitz, "Exercise and older patients: prescribing guidelines," American Family Physician, vol. 74, no. 3, pp. 437-444, 2006.

[23] L. A. Newman, Maintaining Function in Older Adults, Butterworth-Heinemann, Boston, Mass, USA, 1995.

[24] J. C. Nitz and S. R. Hourigan, Physiotherapy Practice in residential Aged Care, Butterworth-Heinemann, Philadelphia, $\mathrm{Pa}, \mathrm{USA}, 2004$. 
[25] J. S. Skinner, Exercise Testing and Exercise Prescription for Special Case: Theoretical Basis and Clinical Application, Lippincott William \& Wilkins, Philadelphia, Pa, USA, 2nd edition, 1993.

[26] A. Zakas, P. Balaska, M. G. Grammatikopolou, N. Zakas, and A. Vergou, "Acute effects of stretching duration on the range of motion of older persons women," Journal of Bodywork and Movement Therapies, vol. 9, pp. 270-277, 2005.

[27] R. E. Rikli and C. J. Jones, "Functional fitness normative scores for community-residing older adults, ages 60-94," Journal of Aging and Physical Activity, vol. 7, no. 2, pp. 162-181, 1999.

[28] P. W. Duncan, D. K. Weiner, J. Chandler, and S. Studenski, "Functional reach: a new clinical measure of balance," Journals of Gerontology, vol. 45, no. 6, pp. M192-M197, 1990.

[29] J. M. VanSwearingen and J. S. Brach, "Making geriatric assessment work: selecting useful measures," Physical Therapy, vol. 81, no. 6, pp. 1233-1253, 2001.

[30] D. Podsiadlo and S. Richardson, "The timed "up and go": a test of basic functional mobility for frail elderly persons," Journal of the American Geriatrics Society, vol. 39, no. 2, pp. 142-146, 1991.

[31] A. D. C. Bastone and W. J. Filho, "Effect of an exercise program on functional performance of institutionalized elderly," Journal of Rehabilitation Research and Development, vol. 41, no. 5, pp. 659-668, 2004.

[32] S. R. Lord, S. Castell, J. Corcoran et al., "The effects of group exercise on physical functioning and falls in frail older people living in retirement villages: a randomized controlled trial," Journal of the American Geriatrics Society, vol. 51, no. 12, pp. 1685-1692, 2003.

[33] O. Seynnes, S. M. A. Fiatarone, O. Hue, P. Pras, P. Legros, and P. L. Bernard, "Physiological and functional responses to lowmoderate versus high-intensity progressive resistance training in frail elders," Journals of Gerontology Series A, vol. 59, no. 5, pp. 503-509, 2004.

[34] J. F. Bean, D. K. Kiely, S. G. Leveille et al., "The 6-minute walk test in mobility-limited elders: what is being measured?" Journals of Gerontology Series A, vol. 57, no. 11, pp. 751-756, 2002.

[35] M. J. Faber, R. J. Bosscher, M. J. Chin, and P. C. van Wieringen, "Effects of exercise programs on falls and mobility in frail and pre-frail older adults: a multicenter randomized controlled trial," Archives of Physical Medicine and Rehabilitation, vol. 87, no. 7, pp. 885-896, 2006.

[36] E. Gillies, T. Aitchison, J. MacDonald, and S. Grant, "Outcomes of a 12-week functional exercise programme for institutionalised elderly people," Physiotherapy, vol. 85, no. 7, pp. 349-357, 1999.

[37] D. A. Lazowski, N. A. Ecclestone, A. M. Myers et al., "A randomized outcome evaluation of group exercise programs in long-term care institutions," Journals of Gerontology Series A, vol. 54, no. 12, pp. 621-628, 1999.

[38] M. E. T. McMurdo and L. M. Rennie, "Improvements in quadriceps strength with regular seated exercise in the institutionalized elderly," Archives of Physical Medicine and Rehabilitation, vol. 75, no. 5, pp. 600-603, 1994.

[39] L. R. Sauvage, B. M. Myklebust, J. Crow-Pan et al., "A clinical trial of strengthening and aerobic exercise to improve gait and balance in elderly male nursing home residents," American Journal of Physical Medicine \& Rehabilitation, vol. 71, no. 6, pp. 333-342, 1992.

[40] H. Littbrand, E. Rosendahl, N. Lindelof, L. Lundin-Olsson, Y. Gustafson, and L. Nyberg, "A high-intensity functional weight-bearing exercise program for older people dependent in activities of daily living and living in residential care facilities: evaluation of the applicability with focus on cognitive function," Physical Therapy, vol. 86, no. 4, pp. 489-498, 2006.

[41] M. J. M. Chin, M. N. M. van Poppel, and W. van Mechelen, "Effects of resistance and functional-skills training on habitual activity and constipation among older adults living in longterm care facilities: a randomized controlled trial," $B M C$ Geriatrics, vol. 6, article 9, 2006.

[42] T. L. Kauffman, O. Jackson, P. Reynolds, J. Barr, and M. Moran, Eds., Geriatric Rehabilitation Manual, Churchill Livingstone, Philadelphia, Pa, USA, 1999.

[43] B. J. Gleberzon and R. S. Annis, "The necessity of strength training for the older patient," The Journal of the Canadian Chiropractic Association, vol. 44, no. 2, pp. 98-102, 2000.

[44] W. D. Bandy, J. M. Irion, and M. Briggler, "The effect of static stretch and dynamic range of motion training on the flexibility of the hamstring muscles," Journal of Orthopaedic and Sports Physical Therapy, vol. 27, no. 4, pp. 295-300, 1998.

[45] J. B. Feland, J. W. Myrer, S. S. Schulthies, G. W. Fellingham, and G. W. Measom, "The effect of duration of stretching of the hamstring muscle group for increasing range of motion in people aged 65 years or older," Physical Therapy, vol. 81, no. 5, pp. 1110-1117, 2001.

[46] American College of Sports Medicine, "Position stand: the recommended quantity and quality of exercise for developing and maintaining cardiorespiratory and muscular fitness, and flexibility in healthy adults," Medicine and Science in Sports and Exercise, vol. 30, no. 6, pp. 975-971, 1998.

[47] E. E. Baum, D. Jarjoura, A. E. Polen, D. Faur, and G. Rutecki, "Effectiveness of a group exercise program in a long-term care facility: a rondomized pilot trial," Journal of the American Medical Directors Association, vol. 4, no. 2, pp. 74-80, 2003.

[48] E. Rydwik, K. Frandin, and G. Akner, "Physical training in institutionalized elderly people with multiple diagnoses; a controlled pilot study," Archives of Gerontology and Geriatrics, vol. 40, no. 1, pp. 29-44, 2005.

[49] S. S. Al-Abdulwahab, "The effects of aging on muscle strength and functional ability of healthy Saudi Arabian males," Annals of Saudi Medicine, vol. 19, no. 3, pp. 211-215, 1999.

[50] J. F. Bean, D. K. Kiely, S. Herman et al., "The relationship between leg power and physical performance in mobilitylimited older people," Journal of the American Geriatrics Society, vol. 50, no. 3, pp. 461-467, 2002.

[51] S. Karinkanta, A. Heinonen, H. Sieranen, K. Uusi-Rasi, and P. Kannus, "Factors predicting dynamic balance and quality of life in home-dwelling elderly women," Gerontology, vol. 51, no. 2, pp. 116-121, 2005.

[52] K. Ringsberg, P. Gerdhem, J. Johansson, and K. J. Obrant, "Is there a relationship between balance, gait performance and muscular strength in 75 years old women?" Journal of Age and Aging, vol. 28, no. 3, pp. 289-293, 1999.

[53] M. E. Daubney and E. G. Culham, "Lower-extremity muscle force and balance performance in adults aged 65 years older," Physical Therapy, vol. 79, no. 12, pp. 1177-1185, 1999. 


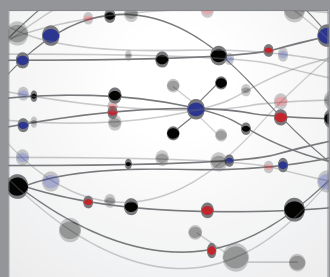

The Scientific World Journal
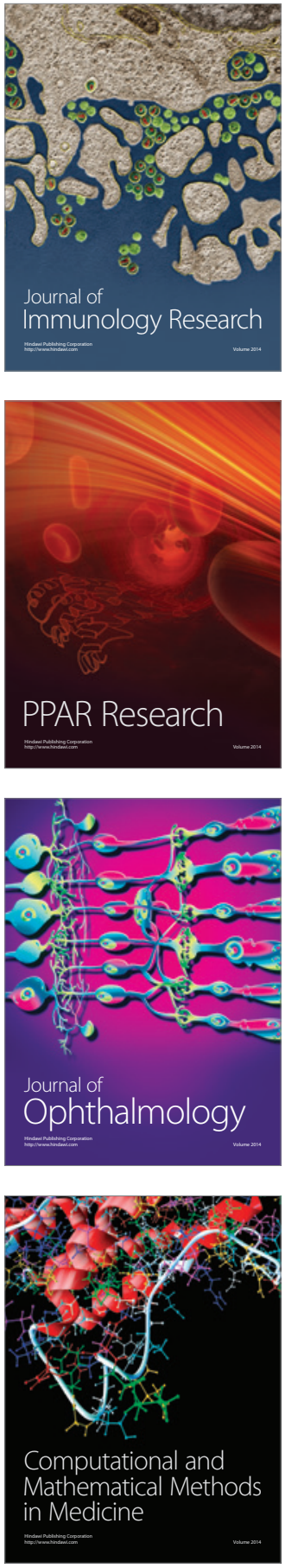

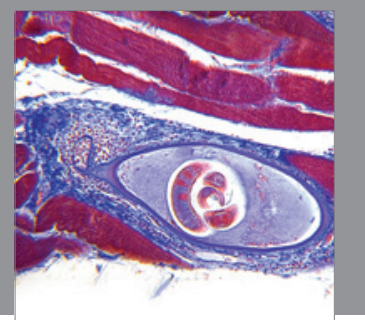

Gastroenterology

Research and Practice
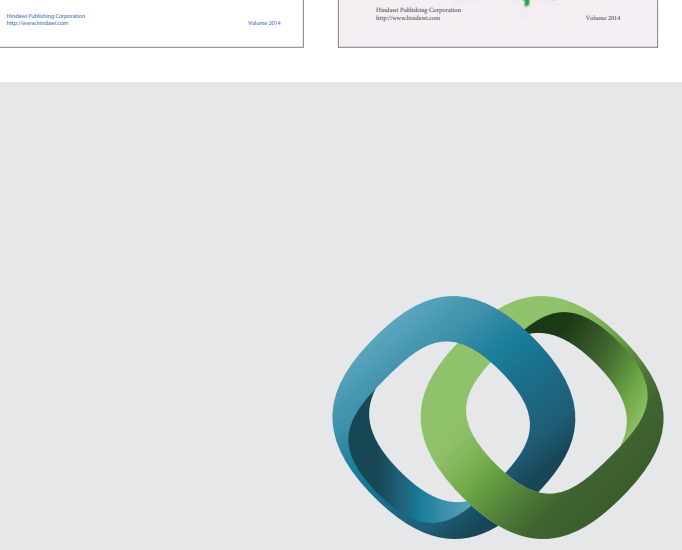

\section{Hindawi}

Submit your manuscripts at

http://www.hindawi.com
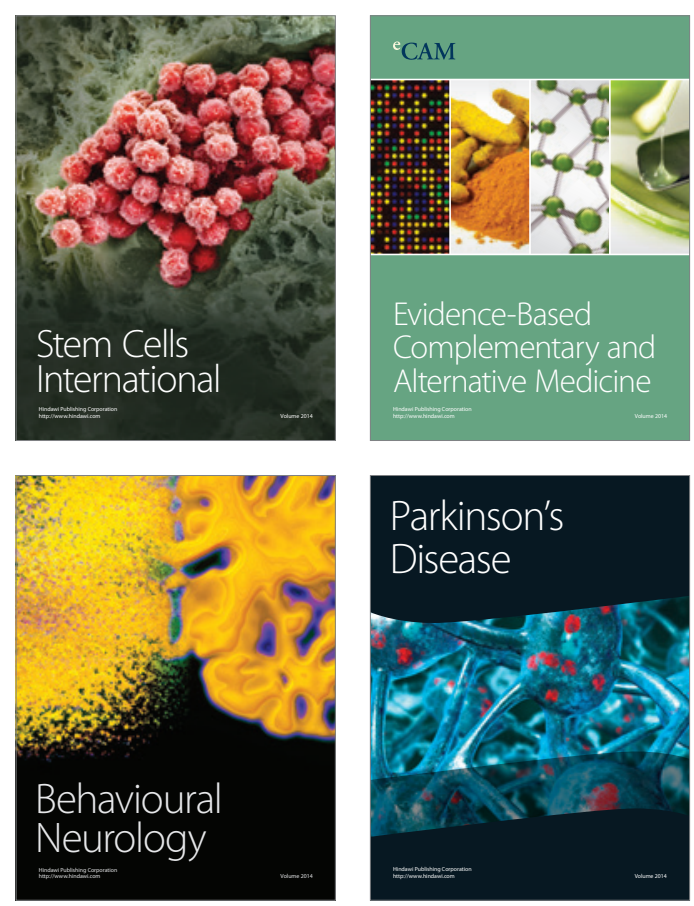

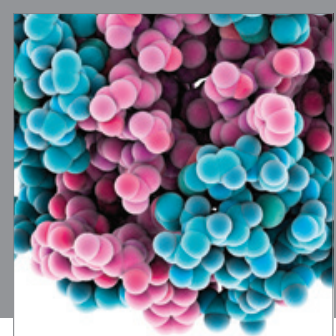

Journal of
Diabetes Research

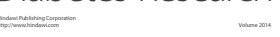

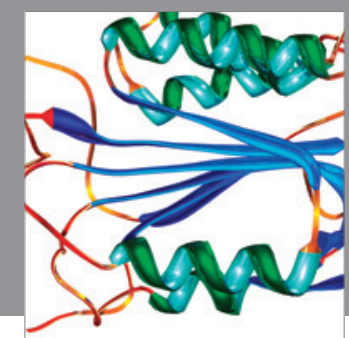

Disease Markers
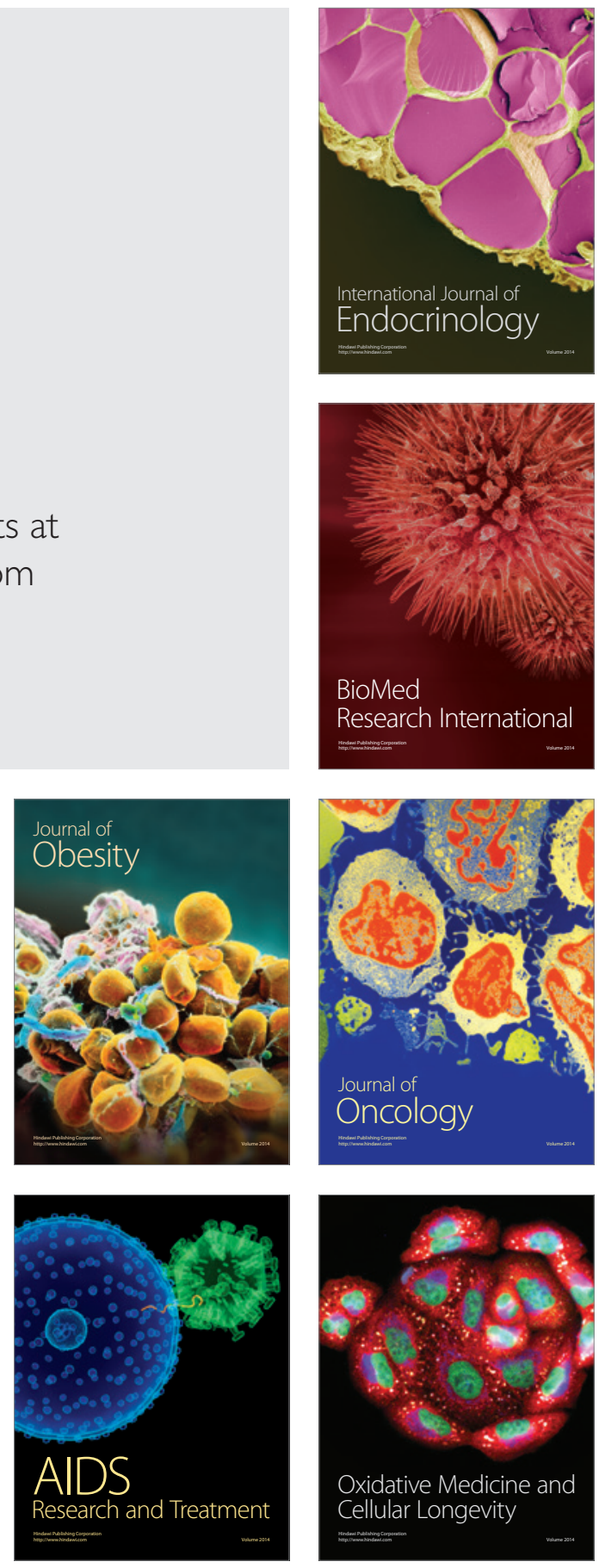